\title{
Arrow's theorem and the Gibbard-Satterthwaite theorem: a unified approach
}

\author{
Philip J. Reny* \\ University of Chicago, Department of Economics, 1126 East 59th Street, Chicago, IL 60637, USA
}

Received 14 January 2000; accepted 27 April 2000

\begin{abstract}
The connection between Arrow's theorem and the Gibbard-Satterthwaite theorem is further strengthened by providing a single proof that yields both results. (C) 2001 Elsevier Science B.V. All rights reserved.
\end{abstract}

Keywords: Arrow's theorem; Gibbard-Satterthwaite theorem

JEL classification: D71

\section{A shared proof}

Let $A$ denote a finite set of alternatives and let $\mathscr{L}$ denote the set of strict linear orders, or (strict) rankings, on $A$. Let $\mathscr{L}^{*}$ denote the set of weak linear orders, or (weak) rankings, on $A$. Fix a positive integer $N$. A function $f: \mathscr{L}^{N} \rightarrow A$ will be called a social choice function, while a function $F: \mathscr{L}^{N} \rightarrow \mathscr{L}^{*}$ will be called a social welfare function. A member of $\mathscr{L}^{N}$ is called a profile of rankings (or simply a profile) and its $i$ th component is called individual $i$ 's ranking. A member of $\mathscr{L}^{*}$ is called a social order, or society's ranking.

We say that a social choice function $f: \mathscr{L}^{N} \rightarrow A$ is:

Pareto Efficient if whenever alternative $a$ is at the top of every individual $i$ 's ranking, $L_{i}$, then $f\left(L_{1}, \ldots, L_{N}\right)=a$.

Monotonic if whenever $f\left(L_{1}, \ldots, L_{N}\right)=a$ and for every individual $i$ and every alternative $b$ the ranking $L_{i}^{\prime}$ ranks $a$ above $b$ if $L_{i}$ does, then $f\left(L_{1}^{\prime} \ldots, L_{N}^{\prime}\right)=a$.

Dictatorial if there is an individual $i$ such that $f\left(L_{1}, \ldots, L_{N}\right)=a$ if and only if $a$ is at the top of $i$ 's ranking $L_{i}$.

*Corresponding author. Tel.: + 1-773-702-8192; fax: +1-773-702-8490.

E-mail address: p-reny@uchicago.edu (P.J. Reny). 
We say that a social welfare function $F: \mathscr{L}^{N} \rightarrow \mathscr{L}^{*}$ is:

Pareto Efficient if whenever alternative $a$ is ranked above $b$ according to each $L_{i}$, then $a$ is ranked above $b$ according to $F\left(L_{1}, \ldots, L_{N}\right)$.

Independent of Irrelevant Alternatives (IIA) if whenever the ranking of $a$ vs. $b$ is unchanged for each $i=1, \ldots, N$ when individual $i$ 's ranking changes from $L_{i}$ to $L_{i}^{\prime}$, then the ranking of $a$ vs. $b$ is the same according to both $F\left(L_{1}, \ldots, L_{N}\right)$ and $F\left(L_{1}^{\prime}, \ldots, L_{N}^{\prime}\right)$.

Dictatorial if there is an individual $i$ such that one alternative is ranked above another according to $F\left(L_{1}, \ldots, L_{N}\right)$ whenever the one is ranked above the other according to $L_{i}$.

In what follows we shall employ essentially a single argument to prove two theorems (Theorems A and B below) ${ }^{1}$. Theorem A is a version of the Muller-Satterthwaite theorem (Muller and Satterthwaite (1977)), and it is well-known that it has as a corollary the Gibbard-Satterthwaite theorem (Gibbard (1973) and Satterthwaite (1975); see Section 2 below. ${ }^{2}$ Theorem B is Arrow's theorem (Arrow, 1963). While the two theorems are known to be closely related, the demonstration below, that effectively a single proof yields both results, indicates that their logical underpinnings are in fact identical. ${ }^{3}$

Of independent interest is that the proof below is both simple and direct. Consequently, Theorem A together with the Proposition in Section 2 provides a simple and direct proof of the GibbardSatterthwaite theorem.

The split-page presentation below is meant to highlight the essentially identical nature of the proofs of Theorems A and B. When reference to a figure is made, the 'social choice' column of the figure applies to the proof of Theorem A, while the 'social order' column of the figure applies to the proof of Theorem B.

Theorem A. If $\# A \geq 3$ and $f: \mathscr{L}^{N} \rightarrow A$ is Pareto efficient and monotonic, then $f$ is a dictatorial social choice function.

\section{Proof}

Step 1. Consider any two distinct alternatives $a, b \in A$ and a profile of rankings in which $a$ is ranked highest and $b$ lowest for every individual $i=1, \ldots, N$. Pareto efficiency implies that the social choice at this profile is $a$.
Theorem B. If $\# A \geq 3$ and $F: \mathscr{L}^{N} \rightarrow \mathscr{L}^{*}$ satisfies Pareto efficiency and IIA, then $F$ is a dictatorial social welfare function.

\section{Proof}

Step 1. Consider any two distinct alternatives $a, b \in A$ and a profile of rankings in which $a$ is ranked highest and $b$ lowest for every individual $i=1, \ldots, N$. Pareto efficiency implies that $a$ is strictly at the top of the social order.

\footnotetext{
${ }^{1}$ Our proof is inspired by the short and elegant proofs of Arrow's theorem due to Geanakoplos (1996).

${ }^{2}$ Anotber corollary of Theorem A is obtained by replacing the hypothesis of monotonicity with Nash implementability. This is because, as Eric Maskin has kindly reminded us, every Nash implementable social choice function is monotonic (see Maskin, 1985).

${ }^{3}$ The alert reader will notice that whenever monotonicity is used in the proof of Theorem A, strategy-proofness (see Section 2) would also have sufficed. With this observation, one obtains side-by-side identical proofs of a version of the Gibbard-Satterthwaite theorem (in which Pareto efficiency replaces the 'onto' assumption) and Arrow's theorem.

${ }^{4}$ The proof in Gibbard (1973) is indirect in that it relies on Arrow's theorem. In contrast, both Satterthwaite (1975) and Schmeidler and Sonnenschein (1978) contain direct proofs. Especially simple direct proofs can be found in Barberà (1983), Benoît (1999a), and Sen (2000). Barberà (1980) and Geanakoplos (1996) contain simple proofs of Arrow's theorem. Also highly recommended is Benoît (1999b), which contains new impossibility results together with simple proofs for social choice correpondences both with and without lotteries.
} 


\begin{tabular}{|c|c|c|c|c|c|c|c|c|}
\hline$L_{1}$ & $\cdots$ & $L_{n-1}$ & $L_{n}$ & $L_{n+1}$ & $\cdots$ & $L_{N}$ & Social Choice & Social Order \\
\hline$b$ & $\cdots$ & $b$ & $a$ & $a$ & $\ldots$ & $a$ & & $a$ \\
\hline$a$ & ... & $a$ & $b$ & . & & . & & . \\
\hline · & & . & . & . & & & $a$ & ; \\
\hline . & & . & . & . & & . & & $b$ \\
\hline . & & . & . & . & & · & & . \\
\hline . & & . & . & $b$ & $\ldots$ & $b$ & & . \\
\hline
\end{tabular}

Fig. 1.

\begin{tabular}{ccccccccc|c}
$L_{1}$ & $\ldots$ & $L_{n-1}$ & $L_{n}$ & $L_{n+1}$ & $\ldots$ & $L_{N}$ & & Social Choice & Social Order \\
$b$ & $\ldots$ & $b$ & $b$ & $a$ & $\ldots$ & $a$ & & $b$ \\
$a$ & $\ldots$ & $a$ & $a$ &. & & $\cdot$ & $\rightarrow$ & $b$ & $a$ \\
$\cdot$ & & $\cdot$ & $\cdot$ & $\cdot$ & &. & & & $\cdot$ \\
$\cdot$ & & $\cdot$ & $\cdot$ & $\cdot$ & &. & & & $\cdot$ \\
$\cdot$ & & $\cdot$ & $\cdot$ & $b$ & $\ldots$ & $b$ & &
\end{tabular}

Fig. 2.

Consider now changing individual 1's ranking by raising $b$ in it one position at a time. By montonicity, the social choice remains equal to $a$ so long as $b$ is below $a$ in 1's ranking. But when $b$ finally does rise above $a$, monotonicity implies that the social choice either changes to $b$ or remains equal to $a$. If the latter occurs, then begin the same process with individual 2 , then 3 , etc. until for some individual $n$, the social choice does change from $a$ to $b$ when $b$ rises above $a$ in $n$ 's ranking. (There must be such an individual $n$ because alternative $b$ will eventually be at the top of every individual's ranking and by Pareto efficiency the social choice will then be $b$.) Figs. 1 and 2 depict the situations just before and just after individual $n$ 's ranking of $b$ is raised above $a$.
Consider now changing individual 1's ranking by raising $b$ in it one position at a time. By IIA, $a$ remains at the top of the social order so long as $b$ is below $a$ in 1 's ranking. But when $b$ finally does rise above $a$, IIA implies that $a$ remains ranked above every alternative but perhaps $b$ by the social order. If $a$ does remain ranked above $b$, then begin the same process with individual 2 , then 3 , etc. until for some individual $n$, the social rank of $b$ rises above $a$ when $b$ rises above $a$ in $n$ 's ranking. (There must be such an individual $n$ because alternative $b$ will eventually be at the top of every individual's ranking and by Pareto efficiency $b$ will then be socially ranked above $a$.) Figs. 1 and 2 depict the situations just before and just after individual $n$ 's ranking of $b$ is raised above $a$.

$$
\begin{array}{cccccccc|c}
L_{1} & \ldots & L_{n-1} & L_{n} & L_{n+1} & \ldots & L_{N} & \text { Social Choice } & \text { Social Order } \\
b & \ldots & b & a & \cdot & & \cdot & & a \\
. & & \cdot & b & \cdot & & \cdot & a & b \\
\cdot & & \cdot & \cdot & \cdot & & . & & \\
\cdot & & \cdot & \cdot & a & \ldots & a & & \cdot \\
a & \ldots & a & . & b & \ldots & b & & \cdot
\end{array}
$$

Fig. 1a. 


\begin{tabular}{|c|c|c|c|c|c|c|c|c|}
\hline $\begin{array}{c}L_{1} \\
b\end{array}$ & $\begin{array}{l}\cdots \\
\ldots\end{array}$ & $\begin{array}{c}L_{n-1} \\
\quad b\end{array}$ & $\begin{array}{c}L_{n} \\
b\end{array}$ & $\begin{array}{c}L_{n+1} \\
.\end{array}$ & $\ldots$ & $L_{N}$ & Social Choice & $\begin{array}{c}\text { Social Order } \\
b\end{array}$ \\
\hline . & & . & $a$ & . & & . & & . \\
\hline . & & . & . & . & & . $\rightarrow$ & $b$ & . \\
\hline . & & . & . & . & & . & & $a$ \\
\hline . & & . & . & $a$ & $\ldots$ & $a$ & & . \\
\hline$a$ & $\ldots$ & $a$ & . & $b$ & $\ldots$ & $b$ & & . \\
\hline
\end{tabular}

Fig. 2a.

Step 2. Consider now Figs. 1a and 2a. Fig. 1a is derived from Fig. 1 (and Fig. 2a from Fig. 2) by moving alternative $a$ to the bottom of individual $i$ 's ranking for $i<n$ and moving it to the second last position in $i$ 's ranking for $i>n$. We wish to argue that these changes do not affect the social choices, i.e., that the social choices are as indicated in the figures.

First, note that the social choice in Fig. 2a must, by monotonicity, be $b$ because the social choice in Fig. 2 is $b$ and no individual's ranking of $b$ vs. any other alternative changes in the move from Fig. 2 to Fig. 2a. Next, note that the profiles in Figs. 1a and 2a differ only in individual $n$ 's ranking of alternatives $a$ and $b$. So, because the social choice in Fig. 2a is $b$, the social choice in Fig. 1a must, by monotonicity, be either $a$ or $b$. But if the social choice in Fig. 1a is $b$, then by monotonicity, the social choice in Fig. 1 must be $b$, a contradiction. Hence, the social choice in Fig. 1a is $a$.

Step 3. Consider $c \in A$ distinct from $a$ and $b$. Because the (otherwise arbitrary) profile of rankings in Fig. 3 can be obtained from the Fig. 1a profile without changing the ranking of $a$ vs.
Step 2. Consider now Figs. 1a and 2a below. Fig. 1a is derived from Fig. $1^{5}$ (and Fig. $2 a^{6}$ from Fig. 2) by moving alternative $a$ to the bottom of individual $i$ 's ranking for $i<n$ and moving it to the second last position in $i$ 's ranking for $i>n$. We wish to argue that these changes do not affect the socially top-ranked alternatives and that the social orders are as indicated in the figures.

First, note that $b$ must, by IIA, be top-ranked by society in Fig. 2a because it is top-ranked in Fig. 2 and no individual's ranking of $b$ vs. any other alternative changes in the move from Fig. 2 to Fig. 2a. Next, note that the profiles in Figs. $1 \mathrm{a}$ and $2 \mathrm{a}$ differ only in individual $n$ 's ranking of alternatives $a$ and $b$. So, by IIA, $b$ must in Fig. 1a remain socially ranked above every alternative but perhaps $a$. But if $b$ is socially ranked at least as high as $a$ in Fig. 1a then by IIA, $b$ would also be socially ranked at least as high as $a$ in Fig. 1, a contradiction. Hence, $a$ is socially ranked first and $b$ second in Fig. 1a.

Step 3. Consider $c \in A$ distinct from $a$ and $b$. Because the (otherwise arbitrary) profile of rankings in Fig. 3 can be obtained from the Fig. 1a profile without changing the ranking of $a$ vs.

\footnotetext{
${ }^{5}$ It can be shown that $b$ is actually ranked second according to the social order. However, it suffices to know that $a$ is top-ranked.

${ }^{6}$ It can be shown that $a$ is actually ranked second according to the social order. However, it suffices to know that $b$ is top-ranked.
} 
any other alternative in any individual's ranking, the social choice in Fig. 3 must, by monotonicity, be $a$.

Step 4. Consider the profile of rankings in Fig. 4 derived from the Fig. 3 profile by interchanging the ranking of alternatives $a$ and $b$ for individuals $i>n$. Because this is the only difference between the profiles in Figs. 3 and 4, and because the social choice in Fig. 3 is $a$, the social choice in Fig. 4 must, by monotonicity, be either $a$ or $b$. But the social choice in Fig. 4 cannot be $b$ because alternative $c$ is ranked above $b$ in every individual's Fig. 4 ranking, and monotonicity would then imply that the social choice would remain $b$ even if $c$ were raised to the top of every individual's ranking, contradicting Pareto efficiency. Hence the social choice in Fig. 4 is $a$.

Step 5. Note that an arbitrary profile of rankings with $a$ at the top of individual $n$ 's ranking can be obtained from the profile in Fig. 4 without reducing the ranking of $a$ vs. any any other alternative in any individual's ranking, society's top-ranked choice in Fig. 3 must, by IIA, be $a$.

Step 4. Consider the profile of rankings in Fig. 4 derived from the Fig. 3 profile by interchanging the ranking of alternatives $a$ and $b$ for individuals $i>n$. Because this is the only difference between the profiles in Figs. 3 and 4, and because $a$ is socially top-ranked in Fig. 3, IIA implies that the social ranking of $a$ remains above $c$ as well as above every other alternative but perhaps $b$ in Fig. 4. But because alternative $c$ is ranked above $b$ in every individual's Fig. 4 ranking, the social ranking of $c$ must be above $b$ by Pareto efficiency. Hence, $a$ is socially topranked and $c$ is socially ranked above $b$ in Fig. 4.

Step 5. Consider an arbitrary profile of rankings with $a$ above $b$ in individual $n$ 's ranking. If necessary, alter the profile by moving alternative $c$ between $a$ and $b$ in $n$ 's ranking and to

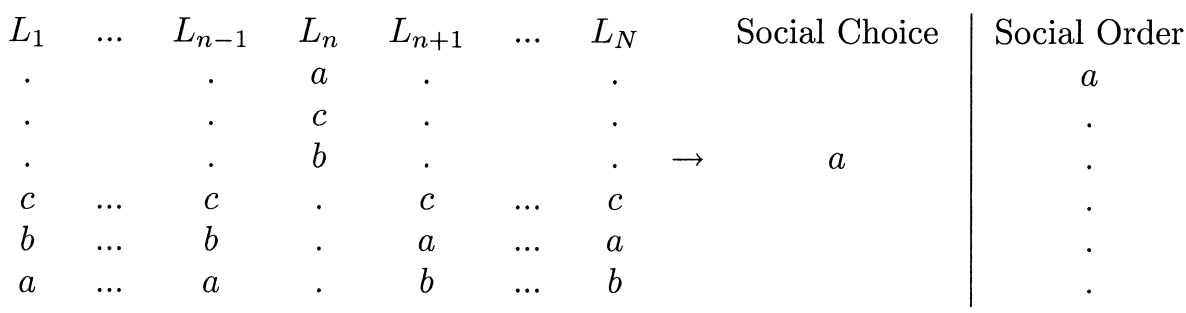

Fig. 3.

\begin{tabular}{|c|c|c|c|c|c|c|c|c|}
\hline$L_{1}$ & $\cdots$ & $L_{n-1}$ & $L_{n}$ & $L_{n+1}$ & ... & $L_{N}$ & Social Choice & Social Order \\
\hline . & & . & $a$ & . & & $\cdot$ & & \\
\hline . & & . & $c$ & . & & . & & - \\
\hline . & & . & $b$ & . & & . & & . \\
\hline . & & $\cdot$ & $\cdot$ & . & & $\cdot$ & $a$ & $c$ \\
\hline$\cdot$ & & · & $\cdot$ & . & & . & & . \\
\hline . & & . & . & . & & . & & . \\
\hline$c$ & $\ldots$ & $c$ & . & $c$ & $\ldots$ & $c$ & & $b$ \\
\hline$b$ & $\ldots$ & $b$ & . & $b$ & $\ldots$ & $b$ & & . \\
\hline$a$ & $\ldots$ & $a$ & . & $a$ & $\ldots$ & $a$ & & . \\
\hline
\end{tabular}

Fig. 4. 
other alternative in any individual's ranking. Hence, monotonicity implies that the social choice must be $a$ whenever $a$ is at the top of individual $n$ 's ranking. So, we may say that individual $n$ is a dictator for alternative $a$. Because $a$ was arbitrary, we have shown that for each alternative $a \in A$, there is a dictator for $a$. But clearly there cannot be distinct dictators for distinct alternatives. Hence there is a single dictator for all alternatives. the top of every other individual's ranking. By IIA this does not affect the social ranking of $a$ vs. $b$. Because the ranking of $a$ vs. $c$ for every individual is now as in Fig. 4, IIA implies that the social ranking of $a$ is above $c$, which by Pareto efficiency is socially ranked above $b$. So, by transitivity, we may conclude that $a$ is socially ranked above $b$ whenever $n$ ranks $a$ above $b$. By repeating the argument with the roles of $b$ and $c$ reversed, and recalling that $c$ was an arbitrary alternative distinct from $a$ and $b$, we may conclude that the social ranking of $a$ is above some alternative whenever $n$ ranks $a$ above that alternative. Thus, we may say that individual $n$ is a dictator for $a$. Since $a$ was an arbitrary alternative we have shown that for every alternative $a \in A$, there is a dictator for $a$. But clearly there cannot be distinct dictators for distinct alternatives. Hence there is a single dictator for all alternatives.

(The procedure used to find the pivotal individual $n$ in step 1 of both proofs is adapted from the ingenious procedure introduced in Geanakoplos (1996). ${ }^{7}$

\section{Gibbard-Satterthwaite}

Recall that a social choice function $f: \mathscr{L}^{N} \rightarrow A$ is strategy-proof if for every individual $i$, every $L \in \mathscr{L}^{N}$, and every $L_{i}^{\prime} \in \mathscr{L}, f\left(L_{i}^{\prime}, L_{-i}\right) \neq f(L)$ implies that $f(L)$ is ranked above $f\left(L_{i}^{\prime}, L_{-i}\right)$ according to $L_{i}$ (and so also that $f\left(L_{i}^{\prime}, L_{-i}\right)$ is ranked above $f(L)$ according to $L_{i}^{\prime}$ ).

The following Proposition and its proof are well known (see Muller and Satterthwaite, 1977, or Mas-Colell et al., 1995). We include them here for completeness.

Proposition. If $f: \mathscr{L}^{N} \rightarrow A$ is strategy-proof and onto, then $f$ is Pareto efficient and monotonic.

Proof. Suppose that $f(L)=a$ and that for every alternative $b$, the ordering $L_{i}^{\prime}$ ranks $a$ above $b$ whenever $L_{i}$ does. We wish to show that $f\left(L_{i}^{\prime}, L_{-i}\right)=a$. If, to the contrary, $f\left(L_{i}^{\prime}, L_{-i}\right)=b \neq a$, then strategy-proofness implies $a=f(L)$ is ranked above $f\left(L_{i}^{\prime}, L_{-i}\right)=b$ according to $L_{i}$. But because the ranking of $a$ does not fall in the move to $L_{i}^{\prime}$, this means that $a=f(L)$ must also be ranked above $b=f\left(L_{i}^{\prime}, L_{-i}\right)$ according to $L_{i}^{\prime}$, contradicting strategy-proofness. Hence, $f\left(L_{i}^{\prime}, L_{-i}\right)=f(L)=a$.

Suppose now that $f(L)=a$ and that for every individual $i$ and every alternative $b$, the ordering $L_{i}^{\prime}$ ranks $a$ above $b$ whenever $L_{i}$ does. Because we can move from $L=\left(L_{1}, \ldots, L_{N}\right)$ to $L^{\prime}=\left(L_{1}^{\prime}, \ldots L_{N}^{\prime}\right)$

\footnotetext{
${ }^{7}$ The sense in which an individual is pivotal here is related to, but distinct from, that employed in the elegant proofs due to Barbera (1980,1983).
} 
by changing the ranking of each individual $i$ from $L_{i}$ to $L_{i}^{\prime}$ one at a time, and because we have shown that the social choice must remain unchanged for every such change, we must have $f\left(L^{\prime}\right)=f(L)$. Hence, $f$ is monotonic.

Choose $a \in A$. Because $f$ is onto, $f(L)=a$ for some $L \in \mathscr{L}^{N}$. By monotonicity the social choice remains equal to $a$ when $a$ is raised to the top of every individual's ranking. But again by monotonicity, the social choice must remain $a$ regardless of how the alternatives below $a$ are ranked by each individual. Consequently, whenever $a$ is at the top of every individual's ranking the social choice is $a$. Because $a$ was arbitrary $f$ is Pareto efficient.

Theorem A and the Proposition together yield the following result.

Corollary. (Gibbard-Satterthwaite) If $\# A \geq 3$ and $f: \mathscr{L}^{N} \rightarrow A$ is onto and strategy-proof, then $f$ is dictatorial.

\section{Acknowledgements}

I wish to thank Vijay Krishna and Motty Perry for motivating the present note through a stimulating discussion of a short proof of Arrow's Theorem due to John Geanakoplos. Thanks also to Drew Fudenberg and Eric Maskin for helpful comments and to Matt Jackson and Roger Myerson for bringing to my attention the work of Muller and Satterthwaite (1977), to Hugo Sonnenschein for directing me to the work of Barberà $(1980,1983)$ and to Mark Satterthwaite for reminding me of the work of Schmeidler and Sonnenschein (1978). Financial support from the the National Science Foundation (SBR-9709392 and SES-9905599) is gratefully acknowledged.

\section{References}

Arrow, K.J., 1963. Social Choice and Individual Values, 2nd Edition. Wiley, New York.

Barberà, A.S., 1980. Pivotal voters: a new proof of Arrow's Theorem. Economics Letters 6, 13-16.

Barberà, A.S., 1983. Strategy-proofness and pivotal voters: a direct proof of the Gibbard-Satterthwaite Theorem. International Economic Review 24, 413-418.

Benoît, J.-P., 1999a. The Gibbard-Satterthwaite Theorem: a simple proof. Mimeo, NYU.

Benô̂t, J.-P., 1999b. Strategyproofness for correspondences and lotteries. Mimeo, NYU.

Geanakoplos, J., 1996. Three brief proofs of Arrow's Impossibility Theorem. Mimeo, Cowles Foundation, Yale University. Gibbard, A., 1973. Manipulation of voting schemes: a general result. Econometrica 41, 587-601.

Mas-Colell, A., Whinston, M.D., Green, J.R., 1995. Microeconomic Theory. Oxford University Press, New York.

Maskin, E., 1985. The theory of implementation in nash equilibrium. In: Hurwicz, L., Schmeidler, D., Sonnenschein, H. (Eds.), Social Goals and Social Organization: Essays in Honor of Elisha A. Panzer. Cambridge University Press, Cambridge.

Muller, E., Satterthwaite, M.A., 1977. The equivalence of strong positive association and strategy-proofness. Journal of Economic Theory 14, 412-418.

Schmeidler, D., Sonnenschein, H., 1978. Two proofs of the Gibbard-Satterthwaite theorem on the possibility of strategyproof social choice functions. In: Gottinger, H.W., Leinfellner, W. (Eds.), Decision Theory and Social Ethics, Issues in Social Choice. D. Reidel Publishing, Dordrecht, Holland, pp. 227-234.

Satterthwaite, M.A., 1975. Strategy-proofness and Arrow's conditions: existence and correspondence theorems for voting procedures and social welfare functions. Journal of Economic Theory 10, 187-217.

Sen, A., 2000. Another direct proof of the Gibbard-Satterthwaite Theorem. Discussion Paper 00-09, Indian Statistical Institute, Delhi Centre, New Delhi. 